\title{
Linear isometries of subspaces of spaces of continuous functions
}

by

W. P. NOVINGER (Tallahassee, Flo.)

Abstract. Let $X$ and $X$ be respectively compact and locally compact Hausdorf spaces, and $A$ be a linear subspace of $O(X)$ which separates points and contains the constant functions. It is shown that if $T$ is a linear isometry of $A$ into $O_{0}(Y)$ and $B=T(A)$, then there are continuous maps $e: \mathrm{Ch}(B) \rightarrow\{z:|z|=1\}$ and $h: \mathrm{Ch}(B) \rightarrow$ $B=T(A)$, then there are continuous maps $e: \operatorname{Ch}(B) \rightarrow\{z:|z|=1\}$ and $h: \mathrm{Ch}(B) \rightarrow$
$\mathrm{Ch}(A)$, such that $T f(y)=e(y) f(h(y))$ for all $f \in A$ and all $y \in \mathrm{Ch}(B)$. Here $\mathrm{Ch}(B)$ and $\mathrm{Ch}(A)$ denote the Choquet boundaries of respectively $A$ and $B$. Results obtained in this paper extend work of W. Holsztyński which appeared in Studia Math. 26 (1966).

Let $C(X)$ and $C(Y)$ denote the familiar supremum normed Banach algebras of continuous complex-valued functions on compact Hausdorff spaces $X$ and $Y$. The well-known Banach-Stone Theorem ([2]; p. 441) states that if $T: C(X) \rightarrow C(Y)$ is a linear isometry of $C(X)$ onto $C(\bar{Y})$, then there is a continuous complex function $e$ of constant modulus 1 and a homeomorphism $h$ of $Y$ onto $X$ such that

$$
T f(y)=e(y) f(h(y)) \quad(f \in O(X)),(y \in Y) .
$$

W. Holsztyński [4] has generalized this theorem to the case of linear isometries of $C(X)$ into $C(Y)$. His conclusion in this case is that there is a closed subset $Y_{0}$ of $Y$, a continuous complex function $e$ of constant modulus 1 on $Y_{0}$, and a continuous surjection $h: Y_{0} \rightarrow X$ such that $(*)$ holds with $Y$ replaced by $Y_{0}$. Holsztyński's result has been applied, for example, by Pelczyniski in his paper [5] and by Baker in [1]. In the present paper we shall go a step further and describe the linear isometries of certain subspaces of $C(X)$ into $O_{0}(Y)$. This description will presently be stated and proved, but first we must record a few additional definitions and facts.

Let $Y$ be a locally compact Hausdorff space, let $C_{0}(Y)$ be the supremum normed Banach algebra of complex-valued continuous functions on $Y$ which vanish at infinity, and let $B$ be a sup-normed linear subspace of $C_{0}(Y)$. The unit ball $V$, in the normed dual $B^{*}$ of $B$, has extreme points and this set of points will be denoted by $\operatorname{ex}(V)$. It is a consequence of Milman's "converse" ([6], p. 9) that each member of ex $(V)$ has the form 
$z \psi_{y}$, where $z$ is a complex number of modulus $1, y \in Y$, and $\psi_{y}$ is the evaluation functional, $\psi_{y}(g)=g(y)(g \in B)$. The Choquet boundary for $B$, denoted by $\mathrm{Ch}(B)$, is defined as $\left\{y \in Y: \psi_{y} \in \operatorname{ex}(V)\right\}$. (Although this definition of $\mathrm{Ch}(B)$ differs from that in [6], p. 38, - Phelps (as do most authors) defines the Choquet boundary only for $Y$ compact and subspaces of $O(Y)$ which contain the constant functions - the two definitions nerertheless agree whenever both are applicable.) A property of the Choquet boundary which we will need later is that it is a "boundary for $B$ " in the sense that each $g \in B$ assumes its maximum absolute value at some point of $\mathrm{Ch}(B)$. Only minor modifications of the proof of Proposition 6.3 in $[6]$, p. 40 , are required to establish this. We are now ready for the main theorem of this paper.

THEOREM 1. Let $X$ and $Y$ be respectively compact and locally compact Hausdorff spaces and $A$ be a linear subspace of $C(X)$ which separates the points of $X$ and contains the constant functions. Suppose that $T$ is a linear isometry of $A$ into $C_{0}(Y)$ and $B=T(A)$. Then there are continuous functions $e$ and $h$ on $Y$ such that $e: \operatorname{Ch}(B) \rightarrow\{z:|z|=1\}, h: \mathrm{Oh}(B) \rightarrow \mathrm{Ch}(A)$ is onto, and such that

$$
T f(y)=e(y) f(h(y)) \quad(f \in A, y \in \mathrm{Ch}(B)) .
$$

Remark. Many of the ideas in the proof to be given below are well known and are to be found, for example, in Hoffman's book [3], pp. 144147. Holsztyński's argument in [4], however, is quite different from the one which we present here.

Proof of the theorem. Let $U$ denote the closed unit ball in the dual $A^{*}$ of $A$ and equip $U$ with the relative weak* topology of $A^{*}$. Let $V$ have a corresponding meaning in $B^{*}$. The map $x \rightarrow \varphi_{x}$, where $\varphi_{x}(f)$ $=f(x)(f \in A)$, is then a homeomorphism of $X$ into $U$ which maps $\operatorname{Ch}(A)$ onto $\operatorname{ex}(K)$, where $K=\left\{\varphi \epsilon A^{*}: \varphi(1)=\|\varphi\|=1\right\}$. Verification of the preceding statement is routine and will not be repeated here. As for the map $y \rightarrow \psi_{y}$, this is a continuous map of $Y$ into $V$ which (by definition) sends $\mathrm{Ch}(B)$ into ex $(V)$. The sets of extreme points of $V$ and $U$ have the following descriptions: $\operatorname{ex}(V)=\left\{z \psi_{y}:|z|=1, y \in \operatorname{Ch}(B)\right\}$, while $\operatorname{ex}(U)$ $=\left\{z \varphi_{x}:|z|=1, x \in \mathrm{Ch}(A)\right\}=\{z \varphi:|z|=1, p \in \operatorname{ex}(K)\}$.

Now the adjoint $T^{*}$ of $T$ is an isometry of $B^{*}$ onto $A^{*}$, is weak* continuous, and takes the extreme points of $V$ onto those of $U$. Conse quently, we have a rule $e: \mathrm{Oh}(B) \rightarrow\{z:|z|=1\}$ where given $y_{\epsilon} \mathrm{Ch}(B)$, $e(y)$ is defined as that complex number of modulus 1 such that $\frac{1}{e(y)} T^{*} \psi_{\nu}$ ex $(\boldsymbol{K})$. As a matter of fact, $e(y)=T_{1}(y)$ when $y \in \mathrm{Ch}(B)$; and so we can use this equation to extend $e$ to a function in $C_{0}(Y)$. Finally, define $h: Y \rightarrow A^{*}$ by $h(y)=\overline{e(y)} T^{*} \psi_{y}(y \in Y)$. Then it is easy to see that $h$ is continuous on $Y$ because $e$ is continuous and because the function $y \rightarrow T^{*} \psi_{y}(y \in Y)$ is the composition of the two continuous maps, $y \rightarrow \psi_{y}$ $(y \in X)$ and $T^{*}: B^{*} \rightarrow A^{*}$. Furthermore, if $y \in \mathrm{Ch}(B)$ and $f \in A$, then $\overline{e(y)} T f(y)=\overline{e(y)} \psi_{y}(T f)=\overline{e(y)} T^{*} \psi_{y}(f)=h(y)(f)$. Remembering that $h(y) \epsilon$ $\operatorname{ex}(K)$ and that $\operatorname{ex}(K)$ can be identified with $\mathrm{Ch}(A)$, we can write $T f(y)=e(y) f(h(y))$. It is not difficult to verify that $h$ maps $\mathrm{Ch}(B)$ onto $\mathrm{ex}(K)$, so the proof of the theorem is complete.

Now let us consider some of the corollaries that result when the subspaces $A$ and/or $B(=T(A))$ are assumed to have various additional properties. It will be convenient to henceforth identify $X$ with its image in $U$ via the homeomorphism $x \rightarrow \varphi_{x}(x \in X)$.

Corollary 1 . Suppose that $T$ maps $A$ onto $C_{0}(Y)$ so that $B=C_{0}(Y)$. Then $\mathrm{Oh}(B)=Y, Y$ is compact, and $h$ is a homeomorphism of $Y$ onto $\mathrm{Ch}(A)$.

Proof. The statement that $\mathrm{Ch}(B)=Y$, of course, means that for each $y \in Y, \psi_{y}$ is an extreme point of the unit ball in $\left(C_{0}(\boldsymbol{Y})\right)^{*}$; but then this latter statement follows readily from the Riesz representation theorem ([8], Theorem 6.19, p. 131). That $Y$ must be compact now follows from the fact that $|e(y)|=1$ for all $y \in \mathrm{Ch}(B)(=Y)$ and that $e \in O_{0}(Y)$. Finally, since $C_{0}(Y)$ separates the points of $Y$, the map $y \rightarrow h(y)$ is a oneto-one continuous map of the compact space $Y$ onto $\mathrm{Ch}(A)$ and thus is a homeomorphism.

Note that if we take $A=C(X)$ in the previous corollary, then $\mathrm{Oh}(A)=X$ and we obtain the Banach-Stone theorem.

CoRothary 2. If $\mathrm{Ch}(A)$ is closed in $X$, then $\mathrm{Ch}(B)$ is closed in $Y$. Furthermore, if $B$ separates the points of $Y$ and $Y$ is compact, then $\mathrm{Ch}(A)$ and $\mathrm{Oh}(B)$ are homeomorphic.

Proof. It is almost immediate from what has already been done that $y \in \mathrm{Oh}(B)$ if and only if $h(y) \in \mathrm{Ch}(A)$. Since $h$ is continuous on $Y$, $\mathrm{Oh}(B)=h^{-1}[\mathrm{Ch}(A)]$ is closed in $Y$. If, in addition, $B$ separates $Y$ and $Y$ is compact, then $h$ is a one-to-one continuous map of the compact subset $\mathrm{Oh}(B)$ onto $\mathrm{Ch}(A)$ and thus is a homeomorphism.

Note here that if $A=O(X)$, then $\mathrm{Ch}(A)=X$ and Corollary 2 is essentially the statement of Holsztyński's results [4].

CoRoridARX 3. If $A$ and $B$ are algebras and if $e=T 1$ is identically 1 on $\mathrm{Oh}(B)$, then $T$ is an algebra isomorphism.

Proof. (Of. [3], p. 14.6.) Let $f, g \in A$ and $y \in \mathrm{Oh}(B)$. Since $f g \in A$, we have by Theorem 1 that $T(f g)(y)=e(y) f(h(y)) g(h(y))$ and hence

$$
\text { (**) } \quad e(y) T(f g)(y)=T f(y) T g(y) \text {. }
$$

Now if $e(y)=1$ for $y \epsilon \mathrm{Oh}(B)$ and if $B$ is an algebra, then the above equation (**) implies that $T(f g)$ and $(T f)(T g)$ are functions in $B$ which agree on the "boundary" $\mathrm{Oh}(B)$. Hence $T(f g)=(T f)(T g)$ and we have proved that $T$ is multiplicative. So $T$ is an algebra isomorphism. 
As a final application, let us indicate how the preceding discussion can be used to describe the isometries of certain Banach spaces of Lipschitz functions. Specifically, let $(X, \varrho)$ be a compact metric space and $\operatorname{Lip}(X, \varrho)$ denote the linear space of complex-valued continuous functions $f$ on $X$ for which $\|f\|_{\mathfrak{e}}<\infty$, where

$$
\|f\|_{e}=\sup \left\{\frac{|f(x)-f(t)|}{\varrho(x, t)}: x, t \in X, x \neq t\right\} .
$$

\section{Constructive function theory and spline systems}

by

$$
\|f\|=\max \left\{\|f\|_{\infty},\|f\|_{\varrho}\right\}, \quad f \in \operatorname{Lip}(X, \varrho),
$$

then $(\operatorname{Lip}(X, \varrho),\|\cdot\|)$ is a Banach space. Its structure had been the subject of considerable study in recent years, and we state below one additional property of this space, which is an easy consequence of work that appears in [7], pp. 1150-1156, and in [9], Theorem 5.1, p. 1397, and of that which we have done above. The proof is omitted altogether.

THeOREM 2. Let $(X, \varrho)$ and $(Y, \delta)$ be compact, connected metric spaces, each of diameter at most 1 . Then a map

$$
T:(\operatorname{Lip}(X, \varrho),\|\cdot\|) \rightarrow(\operatorname{Lip}(Y, \delta),\|\cdot\|)
$$

is a linear isometry onto if and only if there is a metric space isometry $h$ of $(Y, \delta)$ onto $(X, \varrho)$ and a complex number $\alpha$ of modutus 1 such that

$$
T f(y)=\alpha f(h(y)) \quad(f \in \operatorname{Lip}(X, \varrho), y \in X) .
$$

\section{References}

[1] J. W. Baker, The separable projection property, in Approximation theory, Academic Press, New York and London, 1973, pp. 247-250

[2] N. D unford and J. T. Schwartz, Linear Operators, Part I, Interscience, New York, 1958.

[3] K. Hoffman, Banach Spaces of Analytic Funotions, Prentico-Hall, Englewood Cliffs, N. J., 1962.

[4] W. Holsztyński, Oontinuous mappings induced by isometries of spaces of continuous functions, Studia Math. 26 (1966), pp. 133-136.

[5] A. Pełczyński, On $O(S)$-subspaces of separable Banaoh spaces, ibid. 31 (1968), pp. 513-522.

[6] R. R. Phelps, Lectures on Ohoquet's Theorem, Van Nostrand Mathernatioal Studies \# 7, D. Van Nostrand, Princeton, N. J., 1966.

[7] A. K. Roy, Extreme points and linear isometries of the Banaoh space of Lipsohits functions, Canad. J. Math. 20 (1968), pp. 1150-1164.

[8] W. Rudin, Real and Oomplex Analysis, MoGraw-Hill, Now Yoxk, 1966. [9] D. R. Sherbert, Banach algebras of Lipschitz funotions, Pacilic J. Math. 13
(1963), pp. 1387-1399.

FLORIDA STATR UNIVERSITY

TALLAHASSEE, FI. 32306 U.S.A.

\section{Z. CIESIELSKI (Sopot)}

Abstract. Integrating $m+1$ times, $m>-1$, the Haar orthogonal functions we obtain a set od spline functions of order $m$. We complete this set of functions with the monomials $1, \ldots, t^{m}$. Now, applying the Schmidt orthonormalization procedure, we get a complete orthonormal set $\left\{f_{n}^{(m)}, n>-m\right\}$ of splines of order $m$. Differentiating and integrating $k$ times the functions $f_{m}^{(m)}, 0<l_{0}<m+1$, we obtain new systems $\left\{f_{n}^{(m, k)}, n \geqslant k-m\right\}$ and $\left\{g_{n}^{\left(m, l_{c}\right)} n>k-m\right\}$ of splines of order $m-k$ and $m+k$, respec$\left\{f_{n}^{(m, k)}, n \geqslant k-m\right\}$ and $\left\{g_{n}^{(m, k)} n>k-m\right\}$ of splines $\delta$ forder $m-k$ and $m+k$, respec-
tively. All these systems are discussed as bases in various function spaces. The convergence a.e. for functions in $L_{1}$ is proved. The direct and inverse theorems of approximation theory for partial sums corresponding to the expansions with respect to $\left\{f_{n}^{(m)}\right.$, $n>-m\}$ are established. New characterization of the Hölder classes is obtained and the linear isomorphism between these classes and suitable sequence spaces is exhibited.

1. Introduction. In this paper the investigations of spline systems started in the works [4]-[11] are continued. Some of the results were announced in [9].

We are concerned with systems of splines which are bases in various classical function spaces.

In Section 3, partial sums of the spline expansions are treated as singular integrals and the convergence almost everywhere for functions in $I_{1}$ is established. Moreover, the norm and local estimates for the sup of the corresponding partial sums are obtained.

The order of approximation by partial sums of the spline expansjons is established in Theorem 4.1. In Lemma 4.3 it is shown that this order of approximation is the best possible in $W_{1}^{m+2}$ unless the functions are polynomials of degree not exceeding $m+1$. The same order of approximation in the case of periodic functions was established earlier in [21] where suitable interpolating spline bases were constructed. In the case of non-periodic tunctions and of interpolating bases similar results were obtained recentily in [1,2].

The norms of the biorthogonal splines are estimated from above and from below in Section 5. Local estimates for these functions are obtained in Section 6.

Theorem 7.1 establishes the relation between the coefficients of the spline series and the series themselves. 